\title{
FBLN5 is targeted by microRNA-27a-3p and suppresses tumorigenesis and progression in high-grade serous ovarian carcinoma
}

\author{
RONGRONG LI $^{1,2^{*}}$, HUAN WU ${ }^{1,2^{*}}$, HUIYANG JIANG ${ }^{1,2}$, QIUMAN WANG $^{1,2}$, ZHIYUAN DOU $^{1,2}$,

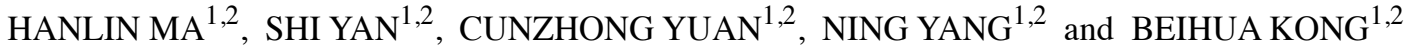 \\ ${ }^{1}$ Department of Obstetrics and Gynecology, ${ }^{2}$ Shandong Key Laboratory of Gynecological Oncology, \\ Qilu Hospital of Shandong University, Jinan, Shandong 250012, P.R. China
}

Received April 23, 2020; Accepted July 31, 2020

DOI: $10.3892 /$ or.2020.7749

\begin{abstract}
High-grade serous ovarian carcinoma (HGSOC) is one of the most lethal gynecological malignancies; however, the precise molecular mechanisms have not been fully characterized. Fibulin-5 (FBLN-5) is an extracellular matrix (ECM) glycoprotein, and plays a crucial role in maintaining the stability of ECM structures, regulating cell proliferation and tumorigenesis. In the present study, the expression of FBLN-5, as determined by western blot analysis and immunohistochemistry, was significantly increased in normal fallopian tube (FT) samples compared with that in HGSOC samples, and decreased FBLN5 expression was associated with unfavorable prognosis of HGSOC. Functional characterization revealed that FBLN5 overexpression significantly inhibited migration, invasion and proliferation abilities of ovarian cancer cells in vitro. Furthermore, micro (mi)RNA-27a-3p (miR-27a-3p) was revealed to be increased in HGSOC, and dual-luciferase reporter assay indicated that miR-27a-3p was functioned as a negative regulator of FBLN5 by directly binding with its 3 '-untranslated region. Collectively, FBLN5 expression was associated with prognosis, proliferation, and metastasis in HGSOC. We hypothesized that FBLN5 was targeted by miR-27a-3p and may serve as a biomarker and provide a new therapeutic approach for the treatment of HGSOC.
\end{abstract}

\section{Introduction}

Ovarian cancer (OC) is one of the leading causes of cancer-associated deaths. There was an estimated 52,100 newly-diagnosed

Correspondence to: Dr Beihua Kong, Department of Obstetrics and Gynecology, Qilu Hospital of Shandong University, 107 Wenhua Xi Road, Jinan, Shandong 250012, P.R. China

E-mail: kongbeihua@sdu.edu.cn

*Contributed equally

Key words: microRNA-27a-3p, fibulin-5, high-grade serous ovarian carcinoma, prognosis, proliferation, metastasis cases and 22,500 fatalities from OC in China in 2015 and 21,750 newly-diagnosed cases and 13,940 fatalities in the USA in 2020 (1,2). High-grade serous ovarian carcinomas (HGSOC) account for $70-80 \%$ of all OC cases and the mortality rate is markedly high (3). Despite the important advances in diagnosis and research, the role and precise mechanisms of HGSOC have not been fully elucidated.

The fibulin protein family widely exists in the extracellular matrix (ECM), and plays a crucial role in the formation and stabilization of the basement membrane, and loose connective tissue and elastic fibers $(4,5)$. In addition to the role in tissue framework, fibulin proteins have also been revealed to be involved in tumorigenesis and progression of cancer (6). Depending on the cell type and cellular context, different fibulins possess both antitumor and pro-tumor properties (7). Fibulin-5 (FBLN5), a 66-kDa secreted glycoprotein, plays significant roles in cell adhesion and motility, and cell-to-cell and cell-to-matrix communication $(8,9)$. In addition, FBLN5 has been revealed to regulate cell growth, cell migration, tissue repair and tumorigenesis (10). Numerous studies have indicated the prognostic potential of FBLN5, as a tumor suppressor in a diverse range of cancers, such as breast cancer, lung cancer and hepatocellular carcinoma (11-14). However, the molecular mechanisms and prognostic significance of FBLN5 in HGSOC have not been characterized.

Micro(mi)RNAs are endogenous, short (21 to 25 nucleotides) non-coding RNAs that repress gene expression at the post-transcriptional level (15). miRNAs target the mRNAs by complementary binding to the homology sequence in the 3'-untranslated region (3'-UTR) (16), and have an overarching regulatory role during carcinogenesis and tumor development (17). Specifically, micro (mi)RNA-27a-3p (miR-27a-3p) was revealed to be a significant positive regulator of tumorigenesis and progression in different types of cancer, including hepatocellular (18), nasopharyngeal (19), and oral squamous carcinoma (20).

In the present study, the expression level of FBLN5 and the molecular mechanisms in patients with HGSOC were elucidated. FBLN5 was identified and proved to negatively regulate the malignant behavior of ovarian cancer both in vitro and in vivo, implying that FBLN5 and its upstream regulator miR-27a-3p has the potential to be a novel therapeutic target of ovarian cancer. 


\section{Materials and methods}

Tissue samples. In the present study, a total of 216 samples [57 normal fallopian tubes (FT) and 159 HGSOC tissues] were collected at Qilu Hospital of Shandong University (Shandong, China) from May 2006 to July 2013. The ages of all included patients ranges from 35 to 78 years. The normal FT samples were obtained from patients who had a benign gynecological tumor, while the HGSOC samples were collected from patients undergoing surgical resection without previous chemotherapy. Written informed consent was provided by each patient prior to surgery. In addition, the guidelines developed by the Ethics Committee of Shandong University Qilu Hospital (KYLL-2018-229) were also adhered to.

Cells culture. The SKOV3 and 293T cell lines were purchased from the American Type Culture Collection and the Chinese Academy of Sciences, respectively. The HEY and A2780 cell lines were a kind gift from the laboratory of Dr. Wei (Department of Gynecology and Obstetrics, Northwestern University, Feinberg School of Medicine). The cells were cultured in the appropriate medium (SKOV3 and A2780 cells, RPMI-1640 medium and HEY and 293T cells, DMEM) (all from Gibco; Thermo Fisher Scientific, Inc.) containing 10\% fetal bovine serum (FBS) (Gibco; Thermo Fisher Scientific, Inc.), and all the cells were maintained in an incubator under standard growth conditions.

RNA extraction and reverse transcription-quantitative PCR $(R T-q P C R)$. Total RNA from cells and samples was extracted using TRIzol ${ }^{\boxplus}$ (Invitrogen; Thermo Fisher Scientific, Inc.). The mRNA and miRNA were reverse-transcribed using the Prime Script RT Reagent kit and One Step Prime Script miRNA cDNA Synthesis kit, respectively, (both from Takara Bio, Inc.) following the manufacturer's guidelines and recommended thermocycling conditions. The cDNA was amplified using the SYBR Green (Takara Bio, Inc.) and Real-Time PCR (qPCR) System (QuantStudio3; Thermo Fisher Scientific, Inc.). The thermocycling conditions were as follows: $95^{\circ} \mathrm{C}$ for $30 \mathrm{sec}$, 40 cycles at $95^{\circ} \mathrm{C}$ for $5 \mathrm{sec}$ and $60^{\circ} \mathrm{C}$ for $30 \mathrm{sec}, 95^{\circ} \mathrm{C}$ for $15 \mathrm{sec}$, $60^{\circ} \mathrm{C}$ for $1 \mathrm{~min}$ and $95^{\circ} \mathrm{C}$ for $15 \mathrm{sec}$. U6 and ACTB were used as the internal controls for normalization and comparison. The $2^{-\Delta \Delta C q}$ method (21) was used to calculate the expression level of the specific genes. The primers are presented in Table SIA.

Western blot analysis. The total protein from the cells and tissues was isolated in ice-cold lysis solution containing RIPA buffer, phenylmethylsulfonyl fluoride and sodium fluoride (100:1:1) (all from Beyotime Bio, Inc.), and then centrifuged for $15 \mathrm{~min}$ at $12,000 \mathrm{x} \mathrm{g}$ at $4^{\circ} \mathrm{C}$. The supernatants were used to calculate the protein concentration using a bicinchoninic acid Assay (Merck KGaA). The proteins $(50 \mu \mathrm{g})$ from each sample were separated using electrophoresis (separation gel, $10-12 \%$; stacking gel, 5\%). Subsequently, the proteins were transferred onto a PVDF membrane $(0.22 \mu \mathrm{m})$, blocked (5\% skim milk for $1 \mathrm{~h}$ at room temperature), and incubated with the primary antibodies overnight at $4^{\circ} \mathrm{C}$. Then the membrane was incubated with the horseradish peroxidase-conjugated secondary antibodies for 1-2 $\mathrm{h}$. Finally, the protein signals were visualized using an enhanced chemiluminescence detection kit (PerkinElmer, Inc.), and the densitometry of the protein bands were analyzed using the Image J v1.8.0 software (National Institutes of Health). GAPDH was used as the endogenous control. The primary antibodies in this study included: Anti-FBLN5 (dilution 1:1,000, cat. no. ab66339; Abcam), anti-GAPDH (dilution 1:1,000, cat. no. 2118; Cell Signaling Technology), anti-N-cadherin (dilution 1:1,000, cat. no. 13116; Cell Signaling Technology), anti-Snail (dilution 1:1,000, cat. no. 3879; Cell Signaling Technology), anti-E-cadherin (dilution 1:1,000, cat. no. 3195; Cell Signaling Technology), anti-p21 (dilution 1:1,000, cat. no. 2947; Cell Signaling Technology), anti-p27 (dilution 1:1,000, cat. no. 3686; Cell Signaling Technology). The secondary antibodies were purchased from KPL: Anti-mouse IgG (cat. no. 5220-0341, dilution 1:6,000) and anti-rabbit IgG (cat. no. 5220-0336, dilution 1:4,000).

Immunohistochemical (IHC) staining. A tissue microarray (TMA) of HGSOC tissues (4- $\mu$ m thick, made by our laboratory) were incubated at $60^{\circ} \mathrm{C}$ for $35 \mathrm{~min}$. The TMAs were immediately deparaffinized, rehydrated with xylene and a graded ethanol series. Subsequently, antigen retrieval was performed using citric acid buffer and microwave irradiation. The non-specific antigens were blocked using a normal goat serum and the slides were then probed with rabbit anti-FBLN5 antibody (1:300; cat. no. ab202977; Abcam) overnight at $4^{\circ} \mathrm{C}$. The signal was detected using 3,3'-diaminobenzidine chromogenic reagent kit (ZSGB Bio, Inc.) and hematoxylin-counterstained (Solarbio Bio, Inc.) according to the manufacturer's instructions. The final score of FBLN5 staining was determined on the basis of extent and intensity.

Plasmid construction and transfection. The coding DNA sequences of FBLN5 were obtained from Shanghai GeneChem Co., Ltd., and then ligated into a pLenti-C-MycDDK-IRES-Puro (PCMV) plasmid (OriGene Technologies, Inc.). Lentivirus expressing FBLN5 were obtained using the 293T cell line packaged with the pMD2.G (Addgene, Inc.) and psPAX2 (Addgene, Inc.) vectors. The FBLN5-expressing stable cells were produced using the infected lentivirus for $24 \mathrm{~h}$ and selected for a week in medium containing antibiotics. Small interfering (si)RNA targeting FBLN5 was synthesized from Shanghai Biosune Biotechnology Co., Ltd., and were similar to those stated in previous studies $(4,22)$. The sequences of the siRNA and miRNA are presented in Table SIB and C.

Cell proliferation assays. The MTT (Sigma-Aldrich; Merck $\mathrm{KGaA}$ ) assay was used to measure cell growth. A total of $(0.8-1) \times 10^{3}$ cells/well were plated in 96 -well plates in sextuplicate, for $1-5$ days. At the same time every day, $20 \mu \mathrm{l}(5 \mathrm{mg} / \mathrm{ml})$ MTT reagent was added to each well, and continued to culture for $4 \mathrm{~h}$ at $37^{\circ} \mathrm{C}$. Subsequently, the supernatant was removed and DMSO (Sigma-Aldrich; Merck KGaA) was then added to each well. The optical density was detected at $490 \mathrm{~nm}$ using a microplate reader (Thermo Fisher Scientific, Inc.). All experiments were performed in triplicate.

Clonogenic assays. To evaluate the colony formation ability, single cells were seeded in a 6 -well plate $(1,000$ cells/well $)$ and cultured for two weeks, under standard culture conditions. 
A
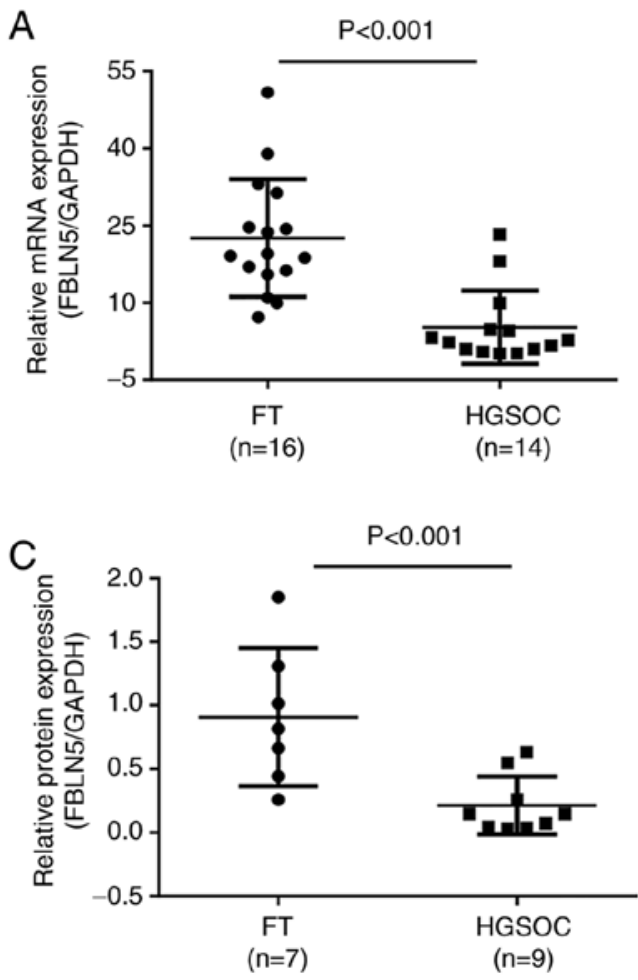

B
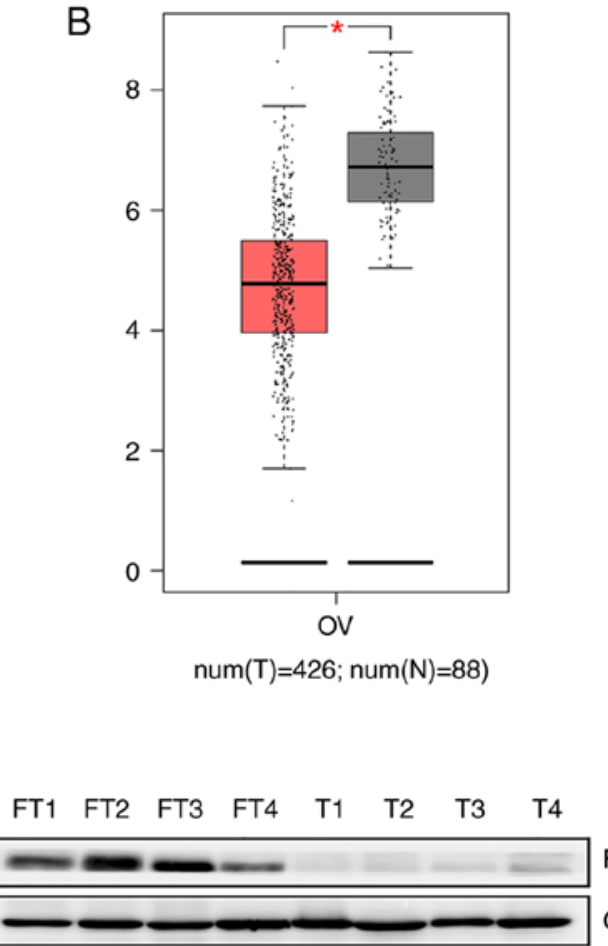

FBLN5

GAPDH

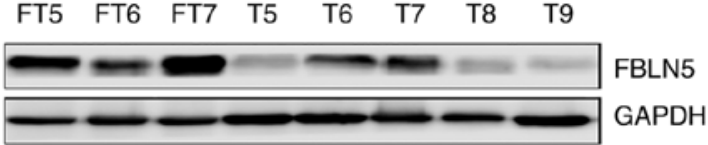

Figure 1. FBLN5 is downregulated in HGSOC tissues. (A) Reverse transcription-quantitative PCR analysis of the FBLN5 mRNA expression level in HGSOC tissues $(n=14)$ and normal FT tissues $(n=16)$. (B) FBLN5 mRNA expression level in ovarian cancer and normal tissues from the GEPIA database. (C) FBLN5 protein expression level of FBLN5 in HGSOC $(n=9)$ and normal FT $(n=7)$ tissues using western blot analysis. ${ }^{*}$ P $<0.05$. FBLN5, fibulin-5; HGSOC, high-grade serous ovarian cancer; T, tumor; FT, fallopian tube.

Thereafter, $100 \%$ methanol was used to fix the colonies for $15 \mathrm{~min}$, and stained with $0.1 \%$ crystal violet for $15 \mathrm{~min}$ at room temperature. Colonies of more than 50 cells were counted. All experiments were performed in triplicate.

Invasion and migration assays. For both the assays, the cells were seeded in a Transwell chamber $(8-\mu \mathrm{m})$, but only the membrane for the invasion assays was coated with Matrigel (both from BD Biosciences). A total of $200 \mu \mathrm{l}$ suspension $\left(1 \times 10^{5}\right.$ cells) was added to the upper chamber, and $700 \mu 1$ medium supplemented with $20 \%$ FBS was added in the lower chamber. Following routine incubation at $37^{\circ} \mathrm{C}$ for the appropriate time-points (6-24h), the cells in the upper chamber were removed using a cotton swab. Subsequently, the cells that reached the lower surface were fixed and stained with $100 \%$ methanol and $0.1 \%$ crystal violet for $15 \mathrm{~min}$ at room temperature, respectively.

Tumor formation assays in nude mice. Female nude mice (BALB/c; 4-5 weeks old; average weight, $15 \mathrm{~g}$ ) were obtained from the NBRI of Nanjing University (Jiangsu, China), and maintained in specific-pathogen-free (SPF) conditions with $25^{\circ} \mathrm{C}$ temperature, $50 \%$ humidity, 12 -h light/dark cycle, and had free access to water and food. Nude mice health and behavior were monitored every day. Then, a $200-\mu 1$ cell suspension $\left(5 \times 10^{6}\right.$ cells) was injected subcutaneously into either side of the armpit, in each nude mouse. After 2-3 weeks, all the mice were euthanized by intraperitoneal injection of pentobarbital sodium $(200 \mathrm{mg} / \mathrm{kg})$, which was confirmed by respiratory and cardiac arrest, and then the tumors were excised and weighed. The diameter of the longest of these tumors was $<2 \mathrm{~cm}$, and all animal experiments adhered to the guidelines and policy of the Shandong University Animal Care and Use Committee.

Bioinformatics analyses. The GEPIA database (http://gepia. cancer-pku.cn/) was used to evaluate the mRNA expression levels of FBLN5 in serous ovarian cancer and normal tissues (23). The TargetScan (http://www.targetscan.org/) and miRanda (http://www.microrna.org) were employed to identify predicted miRNA sequences that may regulate FBLN5 $(24,25)$

Statistical analysis. The data analysis was performed using SPSS v18.0 software (SPSS, Inc.). The unpaired Student's t-test and $\chi^{2}$ test were used to evaluate the association and the significant difference between groups. The curve of overall survival (OS) was assessed using the Kaplan-Meier method and the log-rank test. The experimental data are presented as the mean \pm standard error of the mean and $\mathrm{P}<0.05$ was considered to indicate a statistically significant difference. 
A

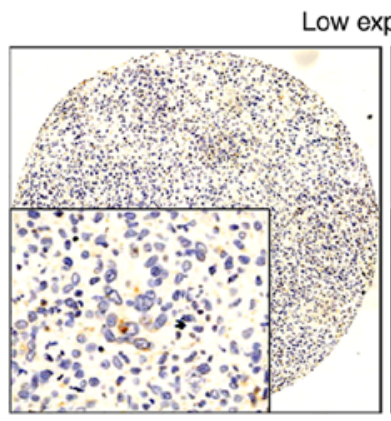

Low expression

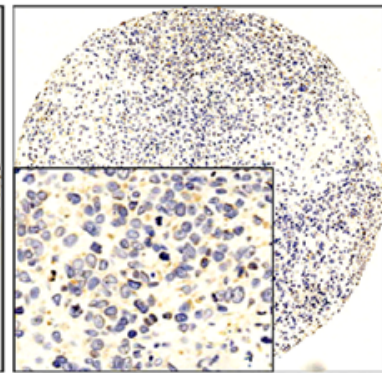

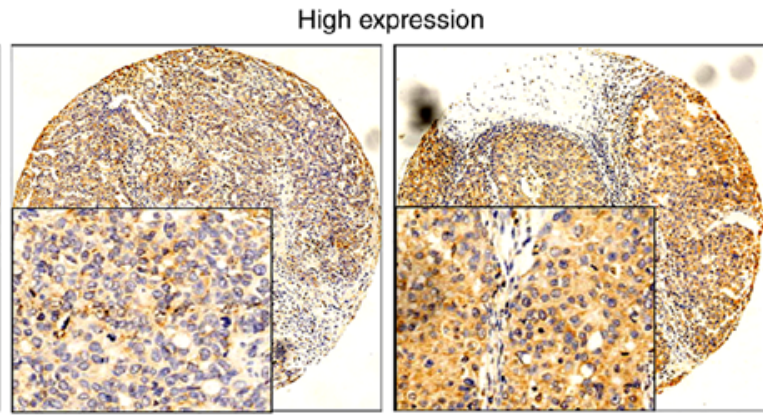

C

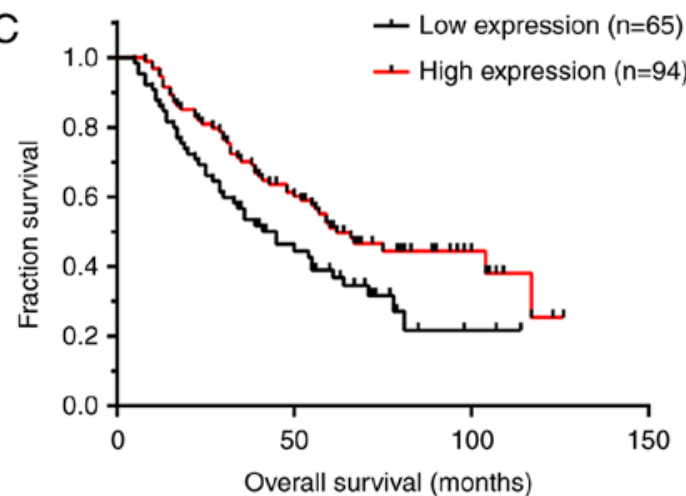

HR $(95 \% \mathrm{Cl}) \quad$ P-value
D

Variables

FBLN5-Low FBLN5-High

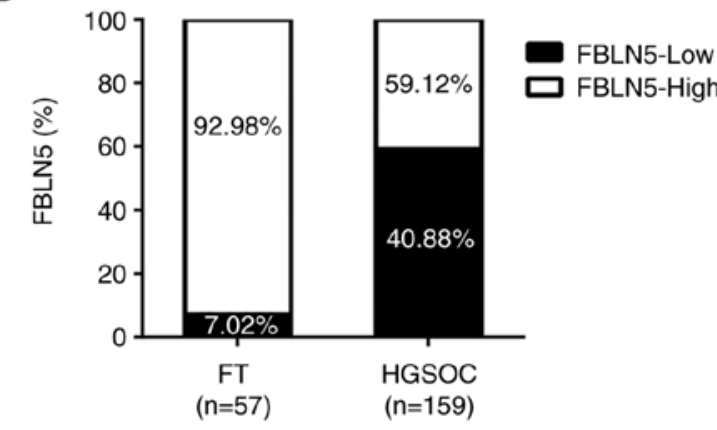

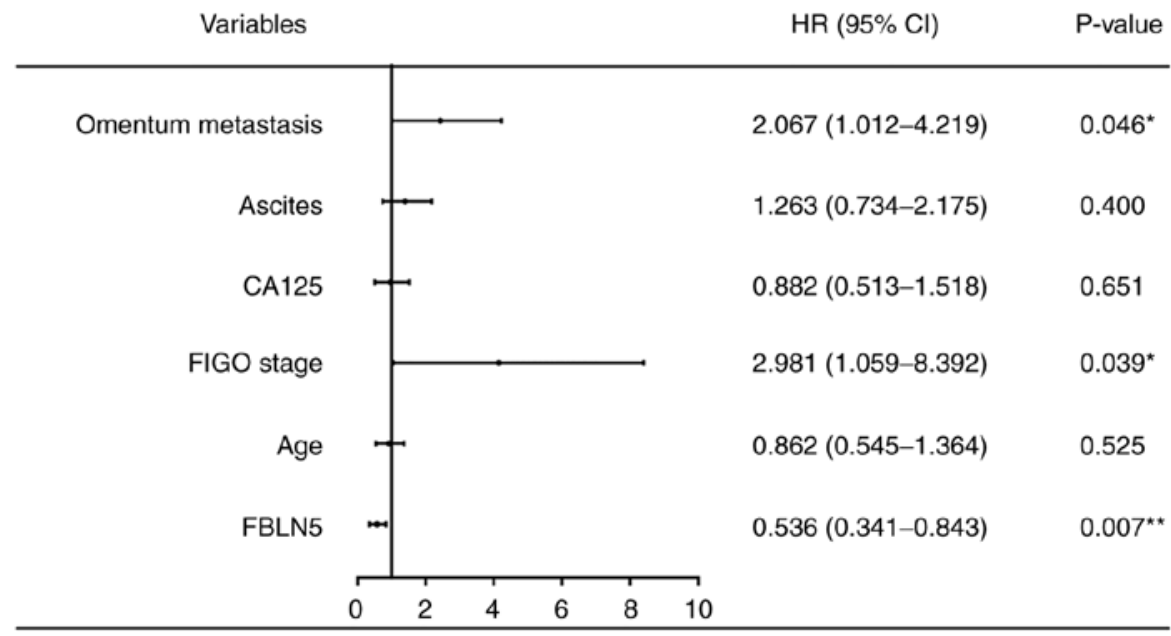

Figure 2. Association of FBLN5 expression with clinical information in patients with HGSOC. (A) Representative immunohistochemical staining of FBLN5 in HGSOC. (B) The high and low expression level of FBLN5 in 57 FT and 159 HGSOC tissues. (C) Overall survival rates in patients with HGSOC, with either high or low expression levels of FBLN5. (D) The multivariate analysis for $\mathrm{OS}$. ${ }^{*} \mathrm{P}<0.05,{ }^{* *} \mathrm{P}<0.01$. FBLN5, fibulin-5; HGSOC, high-grade serous ovarian cancer; FT, fallopian tube.

\section{Results}

FBLN5 expression is significantly downregulated in the HGSOC tissue. The mRNA expression level of FBLN5 in HGSOC and FT tissues (HGSOC, n=14; FT, n=16), was initially investigated and the expression levels of FBLN5 were upregulated in FT tissues compared with that in HGSOC tissues $(\mathrm{P}<0.001$; Fig. 1A). The results from the GEPIA database also revealed that FBLN5 was overexpressed in normal control tissues compared with that in OC samples (Fig. 1B). Then, the protein levels of FBLN5 in FT ( $n=7)$ and HGSOC $(n=9)$ tissue samples were investigated using western blot analysis and were revealed to be significantly different (P<0.001; Fig. 1C).
Low expression level of FBLN5 is associated with unfavorable prognosis. To further investigate the role of FBLN5 in HGSOC, IHC was performed to analyze the protein expression level in a TMA (FT, $\mathrm{n}=57$; HGSOC, $\mathrm{n}=159)$. With respect to the staining degree, the HGSOC tissues were divided into either high $(\mathrm{n}=94)$ and low FBLN5 expression groups $(\mathrm{n}=65)$ (Fig. 2A). There was markedly higher expression levels of FBLN5 in the FT tissues $(92.98 \%$; 53/57) compared with that in the HGSOC tissues (59.12\%; 94/159; Fig. 2B). In addition, the OS of the patients in the FBLN5-high expression group was significantly longer compared with that in patients in the FBLN5-low expression group $(\mathrm{P}<0.05$; Fig. 2C). Using multivariate analysis of the clinicopathological parameters, the data presented in Fig. 2D revealed 
Table I. Association between FBLN5 expression and clinicopathological features.

\begin{tabular}{|c|c|c|c|c|}
\hline \multirow[b]{2}{*}{ Groups } & \multirow[b]{2}{*}{ No. } & \multicolumn{2}{|c|}{$\begin{array}{c}\text { FBLN5 } \\
\text { expression }\end{array}$} & \multirow[b]{2}{*}{ P-value } \\
\hline & & $\begin{array}{l}\text { Low } \\
\text { level }\end{array}$ & $\begin{array}{l}\text { High } \\
\text { level }\end{array}$ & \\
\hline Age (years) & & & & 0.024 \\
\hline$<55$ & 74 & 23 & 51 & \\
\hline$\geq 55$ & 85 & 42 & 43 & \\
\hline FIGO stage & & & & 0.244 \\
\hline $\mathrm{I}+\mathrm{II}$ & 35 & 11 & 24 & \\
\hline III+IV & 120 & 52 & 68 & \\
\hline CA125 (U/ml) & & & & 0.868 \\
\hline$<600$ & 64 & 27 & 37 & \\
\hline$\geq 600$ & 87 & 35 & 52 & \\
\hline Ascites (ml) & & & & 0.502 \\
\hline$<1,000$ & 66 & 24 & 42 & \\
\hline$\geq 1,000$ & 84 & 36 & 48 & \\
\hline Omentum metastasis & & & & 0.040 \\
\hline Negative & 54 & 16 & 38 & \\
\hline Positive & 101 & 48 & 53 & \\
\hline Lymph node metastasis & & & & 0.566 \\
\hline Negative & 41 & 11 & 30 & \\
\hline Positive & 22 & 8 & 14 & \\
\hline Recurrence & & & & 0.008 \\
\hline No & 28 & 5 & 23 & \\
\hline Yes & 97 & 45 & 52 & \\
\hline OS (years) & & & & 0.016 \\
\hline$<48$ & 79 & 40 & 39 & \\
\hline$\geq 48$ & 80 & 25 & 55 & \\
\hline
\end{tabular}

FBLN5, fibulin-5; FIGO, International Federation of Gynecology and Obstetrics; CA125, cancer antigen 125; OS, overall survival.

that OS was significantly associated with FBLN5 expression (HR, 0.536; $\mathrm{P}=0.007)$, FIGO stage $(\mathrm{HR}, 2.981 ; \mathrm{P}=0.039)$ and omentum metastasis (HR, 2.067; $\mathrm{P}=0.046)$. In addition, analysis of the clinicopathological features indicated that FBLN5 expression was associated with age $(\mathrm{P}=0.024)$, omentum metastasis $(\mathrm{P}=0.040)$, recurrence $(\mathrm{P}=0.008)$, and OS $(\mathrm{P}=0.016$; Table I).

FBLN5 inhibits OS cell proliferation. To evaluate the functional role of FBLN5, three cell lines, including SKOV3, A2780 and HEY were transfected stably with FBLN5 overexpression vector, while the SKOV3 cell line was transfected transiently with FBLN5 siRNA. The growth curve analysis demonstrated that upregulation of FBLN5 notably inhibited cell growth compared with that in the control group (Fig. 3A). Furthermore, the clonogenic assays revealed that overexpression of FBLN5 significantly inhibited the clonogenicity efficiency compared with that in the empty vector control group (Fig. 3B). In addition, the HEY cells transfected with PCMV-FBLN5 and PCMV-NC, were subcutaneously injected into the nude mice. As revealed in Fig. 3C and D, FBLN5 overexpression could significantly suppress the growth of OC cell lines in the nude mice. IHC staining of the xenograft tissue was utilized to detect the protein expression level of FBLN5. As demonstrated in Fig. S1, the expression level of FBLN5 in the nucleus was higher in the PCMV-FBLN5 group compared with that in the PCMV-NC group. Lastly, FBLN5 overexpression significantly increased $\mathrm{p} 21$ and $\mathrm{p} 27$ protein expression levels (Fig. 3E). These data indicated that FBLN5 could suppress the proliferation of OC cells.

FBLN5 inhibits OC cell invasion and migration by suppressing epithelial-mesenchymal transition (EMT). To determine the impact of altered FBLN5 expression on the metastasis of the $\mathrm{OC}$ cell lines, the invasion and migration abilities were detected using Transwell and Matrigel assays. As indicated in Fig. 4A and B, FBLN5 downregulation significantly promoted cell invasion and migration abilities. In agreement, overexpression of FBLN5 significantly attenuated the abilities of migration and invasion of the SKOV3, HEY and A2780 cell lines. Then, the molecular mechanism was investigated by analyzing EMT markers. The results revealed that FBLN5 overexpression significantly upregulated an epithelial marker (E-cadherin) and reduced the levels of 2 mesenchymal markers (N-cadherin and Snail) compared with that in the empty vector group, transfected in the HEY, A2780 and SKOV3 cell lines. In addition, FBLN5 knockdown downregulated the epithelial marker (E-cadherin) and increased the expression of 2 mesenchymal markers (N-cadherin, Snail) compared with that in the control group in the SKOV3-transfected cells (Fig. 4C). These data demonstrated that FBLN5 suppressed metastasis of OC cells by inhibiting EMT in vitro.

miR-27a-3p is a direct regulator of FBLN5. Finally, the molecular mechanisms underlying the downregulation of FBLN5 expression in OC was investigated. The potential miRNA that targets the mRNA of FBLN5 was predicted using the miRanda and TargetScan online databases, and miR-27a-3p was selected for further examination. In addition, prior research revealed that miR-27a-3p was upregulated in OC $(26,27)$. As demonstrated in Fig. 5A, the expression level of miR-27a-3p was upregulated in HGSOC compared with that in FT tissues. Further analysis revealed that the expression level of FBLN5 was negatively correlated with miR-27a-3p expression level in HGSOC (Fig. 5B). To further verify the association of direct interaction between miR-27a-3p and FBLN5, the dual-luciferase reporter assay was subsequently performed. The data indicated that upregulation of miR-27a-3p markedly inhibited the luciferase activities in cells co-transfected with wild-type FBLN5 sequences and miR-27a-3p mimics (Fig. 5C). The SKOV3 cell line was transfected with miR-27a-3p inhibitors or mimics. Western blot and RT-qPCR assays demonstrated that miR-27a-3p mimics significantly reduced the protein and mRNA expression levels of FBLN5, respectively, which was reversed by miR-27a-3p inhibitors (Fig. 5D). 


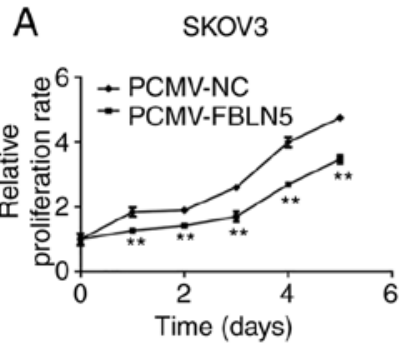

B
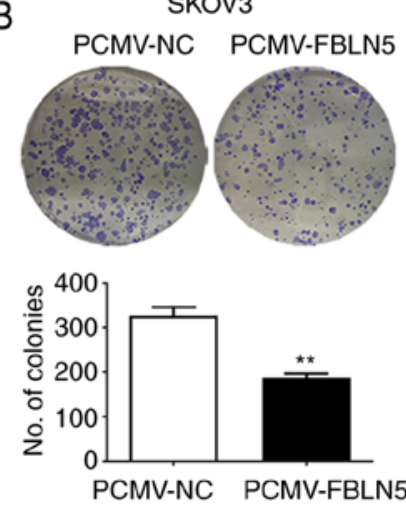

C

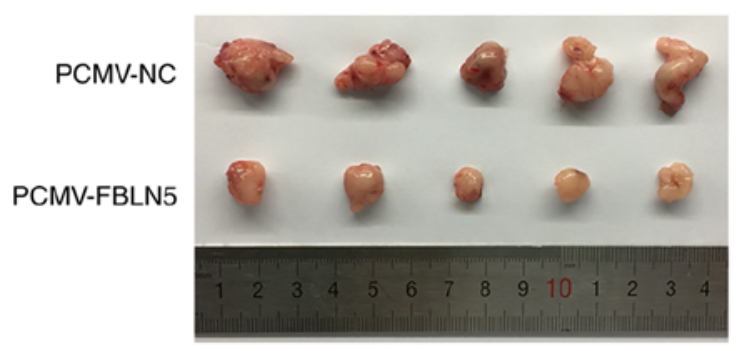

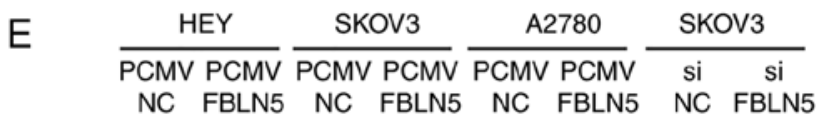
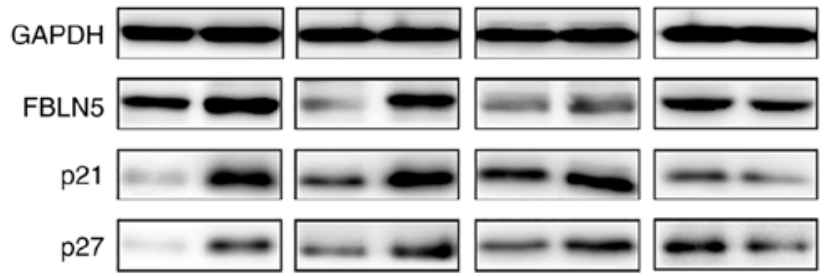
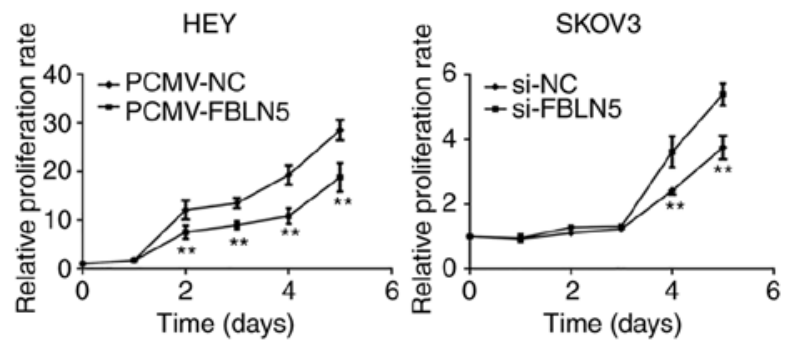

HEY
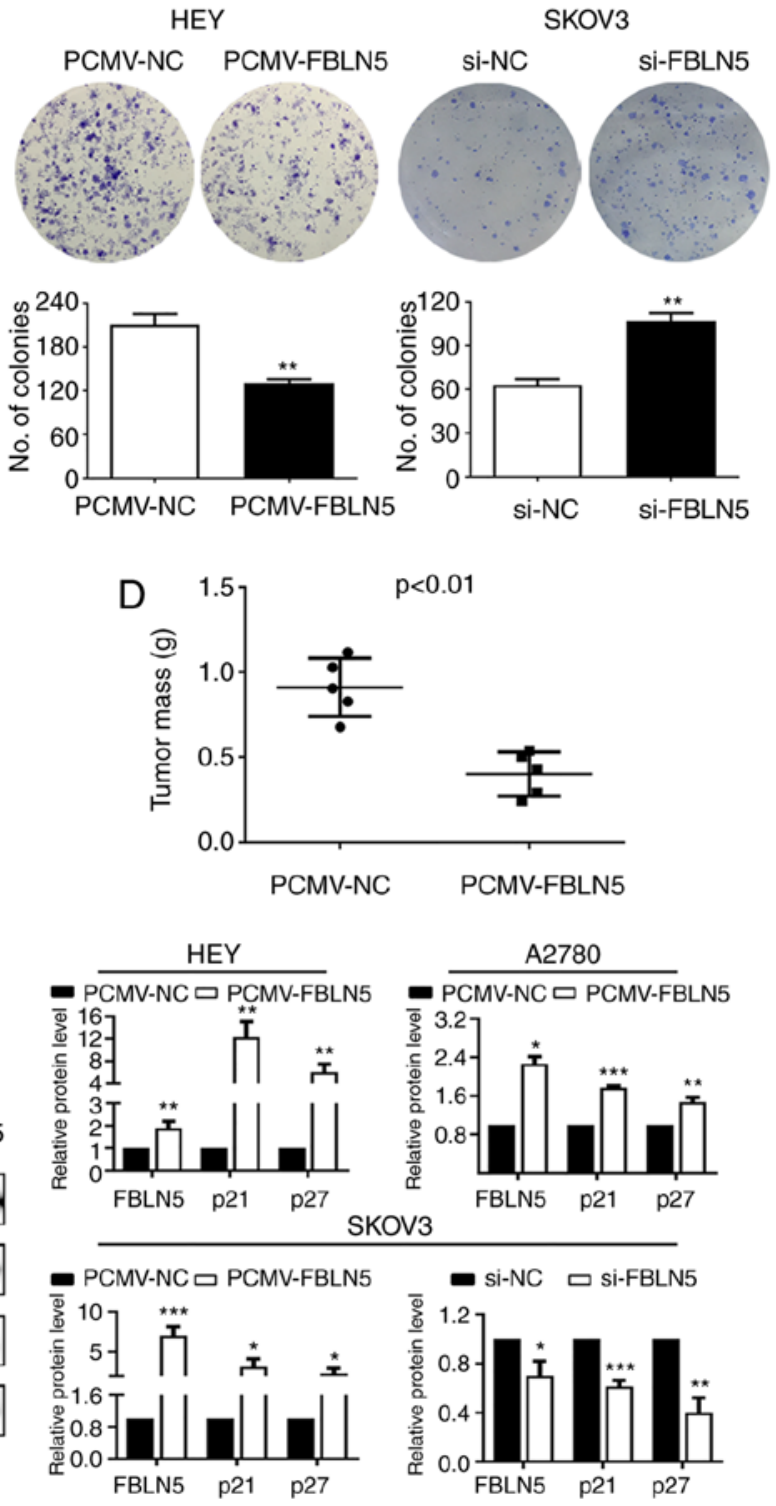

Figure 3. FBLN5 inhibits ovarian cancer cell proliferation in vitro and in vivo. The (A) growth curve and (B) colony formation assay were used to assess the effect of FBLN5 on the proliferation of ovarian cancer cells. (C) The HEY cell line with or without FBLN5 overexpression was injected subcutaneously into nude mice $(n=5)$. (D) The tumor weights in each group were assessed. Data are presented as the mean \pm standard error of the mean. $n=5$. (E) 21 and p27 expression was measured using western blot analysis in ovarian cancer cell lines with FBLN5 overexpression or knockdown. Data are presented as the mean \pm standard error of the mean. $\mathrm{n}=3 .{ }^{*} \mathrm{P}<0.05,{ }^{* *} \mathrm{P}<0.01,{ }^{* * * *} \mathrm{P}<0.001$. FBLN5, fibulin-5; si, small interfering; NC, negative control.

\section{Discussion}

Ovarian carcinoma is the fifth leading cause of cancer-related mortality in females $(28,29)$, and HGSOC is the most malignant type of OC. Most patients with OC are diagnosed at an advanced stage, which leads to poor prognosis $(30,31)$. FBLN5 has been verified to be an anti-oncogene in different types of malignancy (11-14). In the present study, it was revealed that a low expression level of FBLN5 was associated with unfavorable prognosis of HGSOC, providing a potential biomarker for the prediction of diagnosis and prognosis. However, the detailed mechanism of FBLN5 in HGSOC progression remains unknown.

FBLN5 has an anti-proliferative effect in several types of human malignancy (11-13), and FBLN5 overexpression was revealed to suppress DNA synthesis and cyclin A expression in 

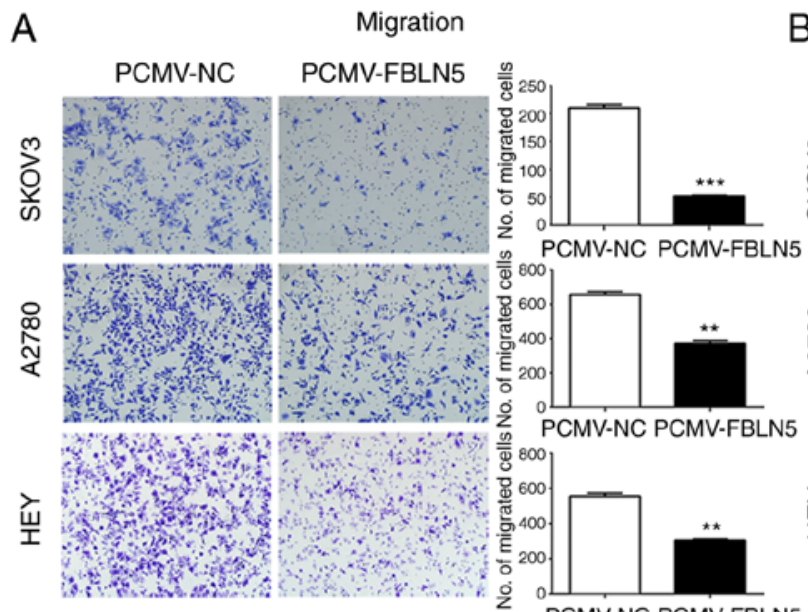

B
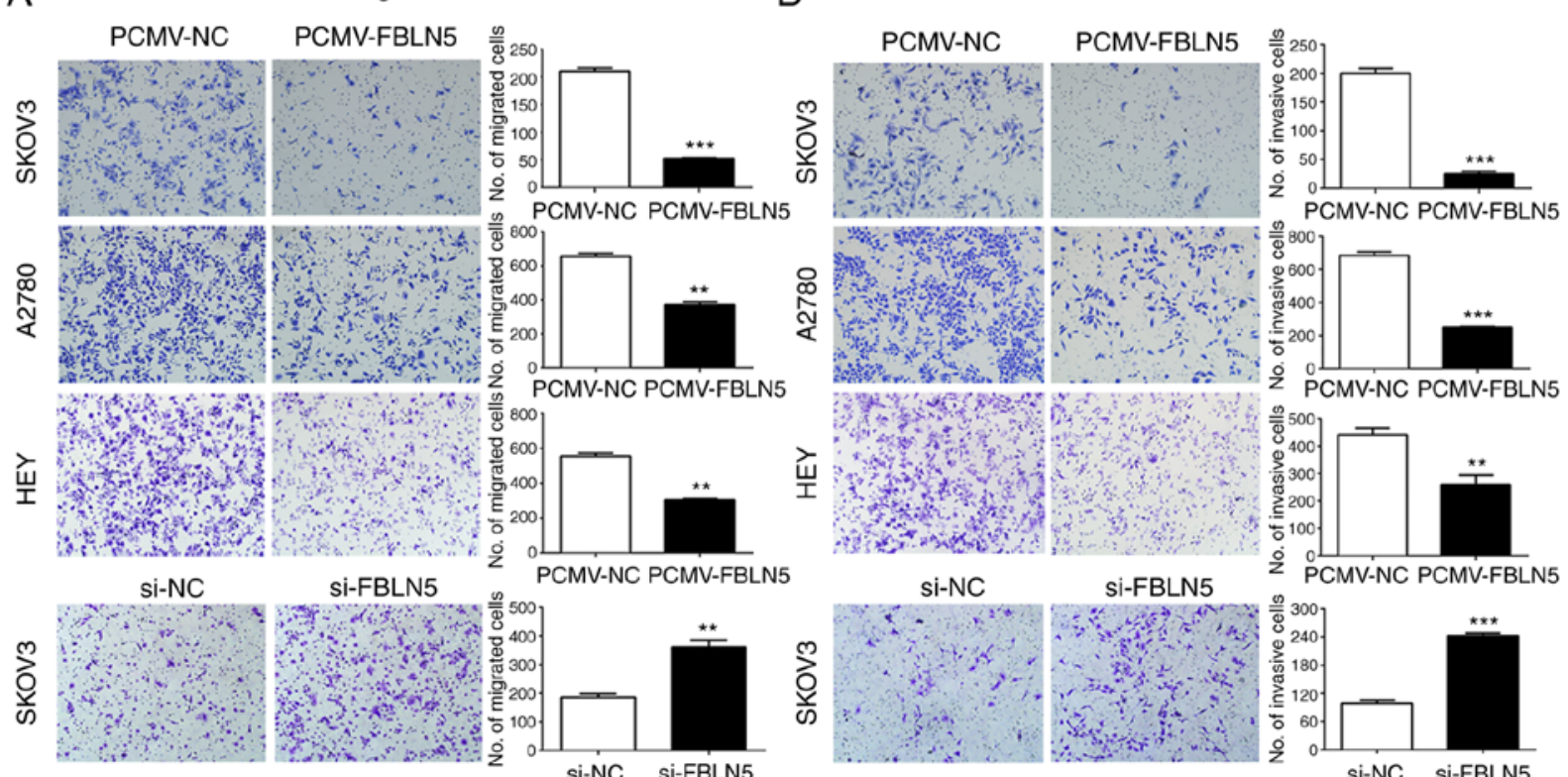

PCMV-NC PCMV-FBLN5
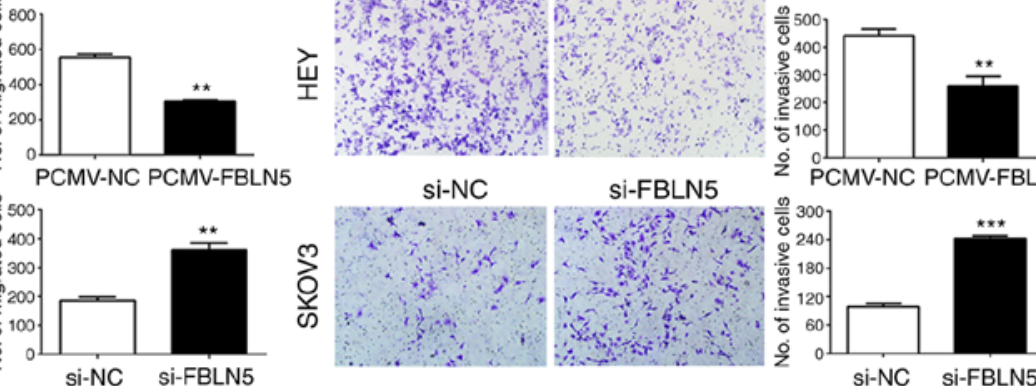

$\stackrel{0}{\text { PCMV-NC PCMV-FBLN5 }}$

C
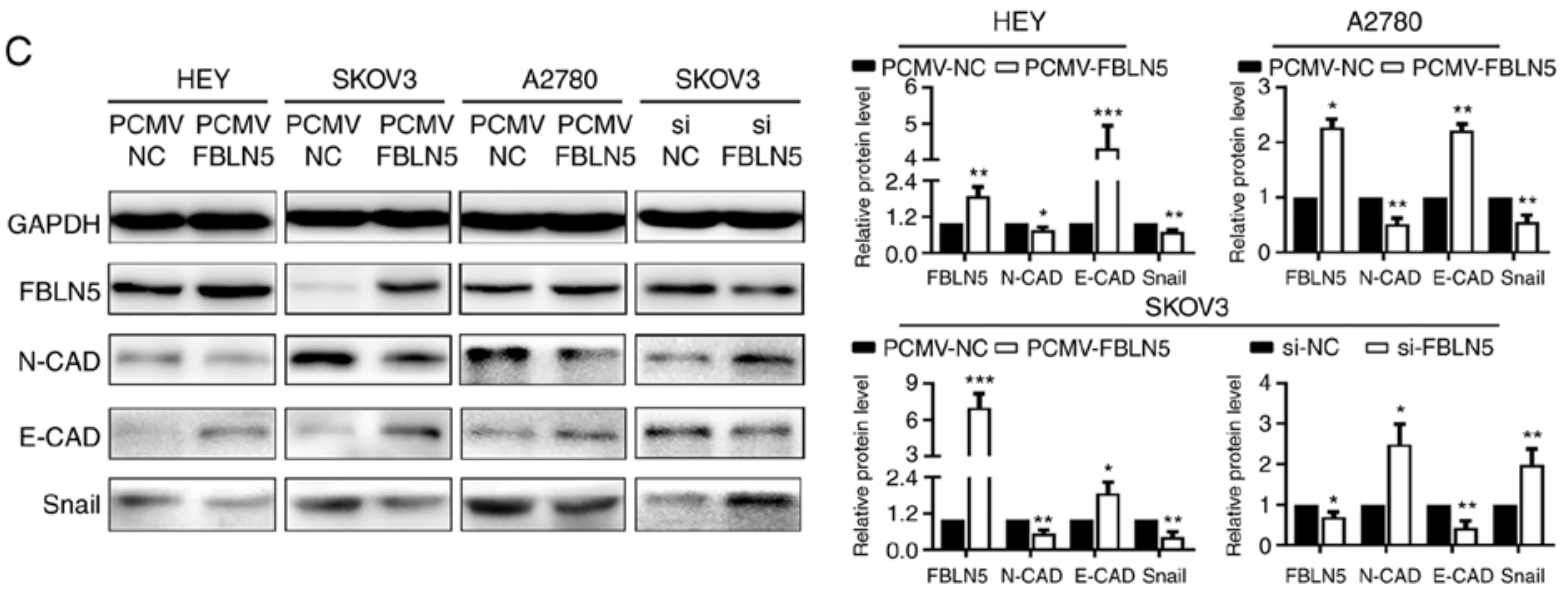

Figure 4. FBLN5 inhibits the migration and invasion of ovarian cancer cells in vitro. Transwell assays were used to assess the effect of FBLN5 overexpression or knockdown on the (A) migration and (B) invasion of ovarian cancer cells. (C) Western blot analysis of the EMT markers, N-cadherin, E-cadherin and Snail. Data are presented as the mean \pm standard error of the mean. $\mathrm{n}=3$. ${ }^{*} \mathrm{P}<0.05,{ }^{* * *} \mathrm{P}<0.01,{ }^{* * * *} \mathrm{P}<0.001$. FBLN5, fibulin-5; EMT, epithelial-mesenchymal transition; si, small interfering; $\mathrm{NC}$, negative control; $\mathrm{N}$-CAD, N-cadherin; E-CAD, E-cadherin.

mink lung epithelial cells (32). p21 and p27 are two important cell cycle-related genes, which regulate cell cycle progression during $G_{1} / S$ and $G_{0} / S$ phases, respectively (33). In the present study, cell proliferation, colony formation and tumor formation assays were performed. Notably, the results revealed that FBLN5 overexpression inhibited OC cell growth in vitro and in vivo, and markedly increased the protein expression level of p21 and p27. Based on these data, we hypothesized that FBLN5 inhibited the proliferation of OC cells by regulating the cell cycle-related proteins. Limitedly, we did not explore the potential relations between FBLN5 and the other essential cell cycle regulators in the present study, such as AURKA, AURKB and FOXM1, which would be carried out relevantly in our future research.

Migration and invasion are important causes of death in patients with cancer. As a vital regulator of metastasis, EMT has been associated with tumor progression, and promotes mobility and resistance to apoptosis in various types of cancer (34). Emerging evidence has revealed that tumor-associated matrix metallopeptidases (MMPs) stimulate EMT (4). Tu et al (13) and Lee et al (35), reported that FBLN5 was involved in EMT and affected the invasion and migration by tumor-associated MMPs in breast cancer and hepatocellular carcinoma cells. EMT is a cellular process and is often defined as the loss of epithelial characteristics and the increase in mesenchymal features $(36,37)$. Consistent with this hypothesis, the results in the present study revealed that FBLN5 overexpression increased the epithelial marker, E-cadherin and decreased the mesenchymal markers, $\mathrm{N}$-cadherin and Snail. Therefore, we hypothesized that FBLN5 inhibited the migration and invasion of OC cells by inhibiting the EMT pathway.

Currently, numerous studies have demonstrated that miRNAs are important regulators in a diverse range of human malignancies (18-20). Furthermore, it has been reported that the function and aberrant expression of miRNAs play a crucial role in several biological processes $(38,39)$. For example, miR-27a-3p promoted cell proliferation in glioma cells by the cooperative 
A

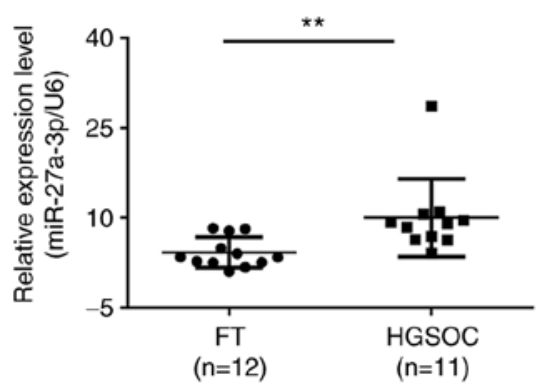

C

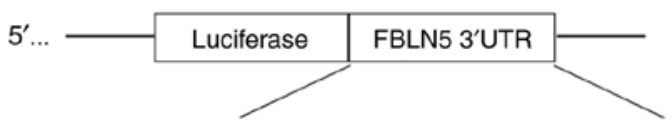

FBLN5 (WT) $\quad 5^{\prime} \ldots$ UCUAUGUUCAAAGACUGUGAA.... $3^{\prime}$ miR-27a-3p $\quad 3^{\prime} . .$. CGCCUUGAAUCGGUGACACUU...5

FBLN5 (MT) $\quad 5^{\prime} .$. UCUAUGUUCAAAGAGUGUGAA... $3^{\prime}$
B
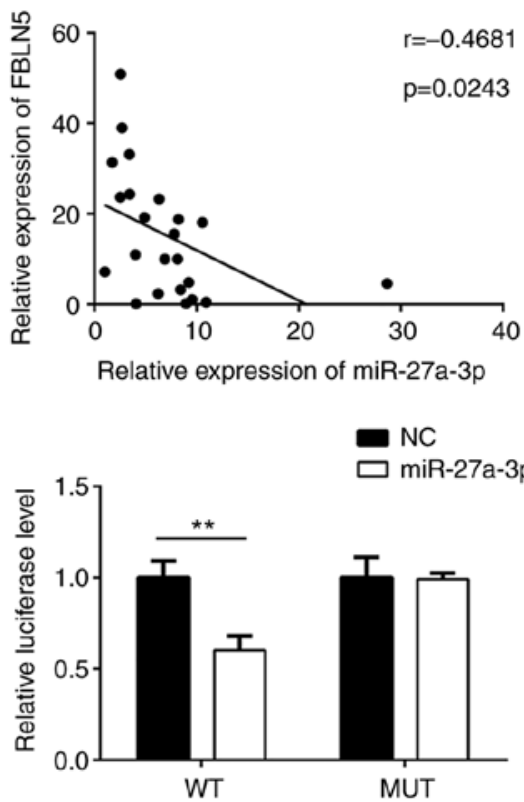

$\mathrm{D}$
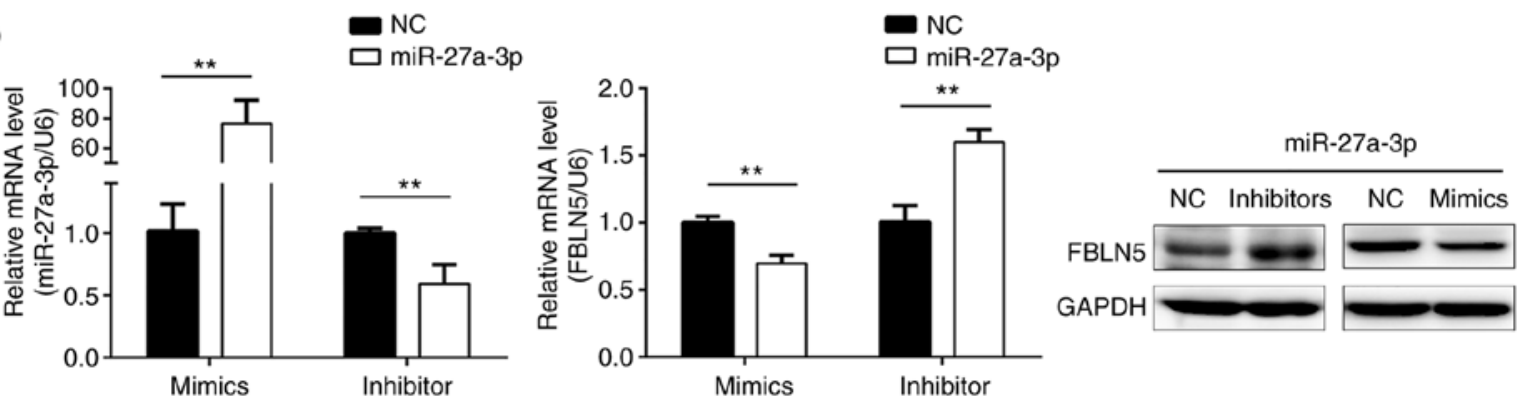

Figure 5. FBLN5 is the target gene of miR-27a-3p. (A) The mRNA level of miR-27a-3p was higher in HGSOC tissues compared with that in FT tissues, using RT-qPCR. (B) Correlation analysis of FBLN5 and miR-27a-3p expression levels. (C) Prediction of the binding sites between miR-27a-3p and 3'-UTR of FBLN5. The binding sequence of miR-27a-3p with the FBLN5 3'UTR sequence was deleted in the mutated type. Dual-luciferase reporter assays were used to analyzed the luciferase activity of the WT or MT FBLN5 3'UTR. (D) The mRNA expression level of miR-27a-3p was detected using RT-qPCR following SKOV3 cell transfection with miR-27a-3p mimics or inhibitor. RT-qPCR and western blot analysis revealed an inverse association between FBLN5 and miR-27a-3p expression. Upregulation of miR-27a-3p inhibited FBLN5 expression level and downregulated miR-27a-3p expression level could promote the expression level of FBLN5. Data are presented as the mean \pm standard error of the mean. ${ }^{* *} \mathrm{P}<0.01$. FBLN5, fibulin-5; miR-27-3p, microRNA-27-3-p; HGSOC, high-grade serous ovarian cancer; FT, fallopian tube; RT-qPCR, reverse transcription-quantitative PCR; UTR, untranslated region; WT, wild-type; MT, mutant; NC, negative control.

regulation of MXI1 (40). Li et al (26) and Wang et al (27), reported that the miR-27a-3p expression level was increased in $\mathrm{OC}$, and primarily affected the growth and metastasis of cancer cells. Consistent with these previous studies, the results from the present study indicated that miR-27a-3p was upregulated in the tissue from patients with HGSOC. In addition, it was verified that FBLN5 was the target of miR-27a-3p, and the miR-27a-3p expression level was negatively correlated with FBLN5 expression level. Therefore, the increased miR-27a-3p expression level could explain why FBLN5 was downregulated in HGSOC.

In summary, the present study indicated that FBLN5 was targeted by miR-27a-3p and suppressed cell proliferation, invasion and metastasis in OC cells. Furthermore, FBLN5 downregulation was associated with unfavorable prognosis. These findings revealed that increasing FBLN5 expression could be a potential new strategy for the treatment of HGSOC.

\section{Acknowledgements}

Not applicable.

\section{Funding}

The present study was supported by the National Natural Science Foundation of China (grant nos. 81572554 and 81874107), and the Science Foundation of Qilu Hospital of Shandong University (grant no. 2019QLQN03).

\section{Availability of data and materials}

The datasets used and/or analyzed during the present study are available from the corresponding author upon reasonable request.

\section{Authors' contributions}

RL and HW designed and conceived the experiments, and wrote the original draft of the manuscript. HJ, QW and ZD collected and analyzed the data. HM, CY and SY provided technical support and revised the manuscript. NY assisted with acquisition and analysis of clinical information. BK directed 
the project and interpreted the results. All authors have read and approved the manuscript.

\section{Ethics approval and consent to participate}

This study was approved by the Shandong University Qilu Hospital Ethics Committee and Shandong University Animal Care and Use Committee (Shandong, China).

\section{Patient consent for publication}

Not applicable.

\section{Competing interests}

All authors declare that they have no competing interests.

\section{References}

1. Chen W, Zheng R, Baade PD, Zhang S, Zeng H, Bray F, Jemal A Yu XQ and He J: Cancer statistics in China, 2015. CA Cancer J Clin 66: 115-132, 2016.

2. Siegel RL, Miller KD and Jemal A: Cancer statistics, 2020. CA Cancer J Clin 70: 7-30, 2020.

3. Bowtell DD, Böhm S, Ahmed AA, Aspuria PJ, Bast RC Jr, Beral V, Berek JS, Birrer MJ, Blagden S, Bookman MA, et al: Rethinking ovarian cancer II: Reducing mortality from high-grade serous ovarian cancer. Nat Rev Cancer 15: 668-679, 2015.

4. Heo JH, Song JY, Jeong JY, Kim G, Kim TH, Kang H, Kwon AY and An HJ: Fibulin-5 is a tumour suppressor inhibiting cell migration and invasion in ovarian cancer. J Clin Pathol 69: 109-116, 2016.

5. Timpl R, Sasaki T, Kostka G and Chu ML: Fibulins: A versatile family of extracellular matrix proteins. Nat Rev Mol Cell Biol 4 479-489, 2003

6. Gallagher WM, Currid CA and Whelan LC: Fibulins and cancer: Friend or foe? Trends Mol Med 11: 336-340, 2005.

7. Obaya AJ, Rua S, Moncada-Pazos A and Cal S: The dual role of fibulins in tumorigenesis. Cancer Lett 325: 132-138, 2012.

8. Yanagisawa H, Schluterman MK and Brekken RA: Fibulin-5, an integrin-binding matricellular protein: Its function in development and disease. J Cell Commun Signal 3: 337-347, 2009.

9. De Vega S, Iwamoto T and Yamada Y: Fibulins: Multiple roles in matrix structures and tissue functions. Cell Mol Life Sci 66: 1890-1902, 2009.

10. Albig AR and Schiemann WP: Fibulin-5 function during tumorigenesis. Future Oncol 1: 23-35, 2005.

11. Chen X, Song X, Yue W, Chen D, Yu J, Yao Z and Zhang L: Fibulin-5 inhibits Wnt/ $\beta$-catenin signaling in lung cancer. Oncotarget 6: 15022,2015.

12. Mohamedi Y, Fontanil T, Solares L, Garcia-Suárez O, GarcíaPiqueras J, Vega JA, Cal S and Obaya AJ: Fibulin-5 downregulates $\mathrm{Ki}-67$ and inhibits proliferation and invasion of breast cancer cells. Int J Oncol 48: 1447-1456, 2016.

13. Tu K, Dou C, Zheng X, Li C, Yang W, Yao Y and Liu Q: Fibulin-5 inhibits hepatocellular carcinoma cell migration and invasion by down-regulating matrix metalloproteinase-7 expression. BMC Cancer 14: 938, 2014.

14. Yue W, Sun Q, Landreneau R, Wu C, Siegfried JM, Yu J and Zhang L: Fibulin-5 suppresses lung cancer invasion by inhibiting matrix metalloproteinase-7 expression. Cancer Res 69: 6339-6346, 2009.

15. Suzuki HI, Yamagata K, Sugimoto K, Iwamoto T, Kato S and Miyazono K: Modulation of microRNA processing by $\mathrm{p} 53$. Nature 460: 529-533, 2009.

16. Ranganathan $\mathrm{K}$ and Sivasankar V: MicroRNAs-biology and clinical applications. J Oral Maxillofac Pathol 18: 229-234, 2014

17. Svoronos AA, Engelman DM and Slack FJ: OncomiR or tumor suppressor? The duplicity of microRNAs in cancer. Cancer Res 76: 3666-3670, 2016.

18. Guo D, Li Y, Chen Y, Zhang D, Wang X, Lu G, Ren M, Lu X and He S: DANCR promotes HCC progression and regulates EMT by sponging miR-27a-3p via ROCK1/LIMK1/COFILIN1 pathway. Cell Prolif 52: e12628, 2019.
19. Li L and Luo Z: Dysregulated miR-27a-3p promotes nasopharyngeal carcinoma cell proliferation and migration by targeting Mapk10. Oncol Rep 37: 2679-2687, 2017.

20. Zeng G, Xun W, Wei K, Yang Y and Shen H: MicroRNA-27a-3p regulates epithelial to mesenchymal transition via targeting YAP1 in oral squamous cell carcinoma cells. Oncol Rep 36: 1475-1482, 2016.

21. Livak KJ and Schmittgen TD: Analysis of relative gene expression data using real-time quantitative PCR and the 2(-Delta Delta C(T)) method. Methods 25: 402-408, 2001.

22. Yamauchi Y, Tsuruga E, Nakashima K, Sawa Y and Ishikawa H: Fibulin-4 and-5, but not fibulin-2, are associated with tropoelastin deposition in elastin-producing cell culture. Acta Histochem Cytochem 43: 131-138, 2010.

23. Tang Z, Li C, Kang B, Gao G, Li C and Zhang Z: GEPIA: A web server for cancer and normal gene expression profiling and interactive analyses. Nucleic Acids Res 45 (W1): W98-W102, 2017.

24. Agarwal V, Bell GW, Nam JW and Bartel DP: Predicting effective microRNA target sites in mammalian mRNAs. Elife 4: e05005, 2015.

25. Betel D, Wilson M, Gabow A, Marks DS and Sander C: The microRNA.org resource: Targets and expression. Nucleic Acids Res 36 (Database Issue): D149-D153, 2008.

26. Li E, Han K and Zhou X: microRNA-27a-3p down-regulation inhibits malignant biological behaviors of ovarian cancer by targeting BTG1. Open Med (Wars) 14: 577-585, 2019.

27. Wang Z, Ji G, Wu Q, Feng S, Zhao Y, Cao Z and Tao C: Integrated microarray meta-analysis identifies miRNA-27a as an oncogene in ovarian cancer by inhibiting FOXO1. Life Sci 210: 263-270, 2018.

28. Armstrong DK, Bundy B, Wenzel L, Huang HQ, Baergen R, Lele S, Copeland LJ, Walker JL and Burger RA; Gynecologic Oncology Group: Intraperitoneal cisplatin and paclitaxel in ovarian cancer. N Engl J Med 354: 34-43, 2006.

29. Chan JK, Cheung MK, Husain A, Teng NN, West D, Whittemore AS, Berek JS and Osann K: Patterns and progress in ovarian cancer over 14 years. Obstet Gynecol 108: 521-528, 2006.

30. Kurman RJ: Origin and molecular pathogenesis of ovarian highgrade serous carcinoma. Ann Oncol 24 (Suppl 10): x16-x21, 2013.

31. Mezzanzanica D: Ovarian cancer: A molecularly insidious disease. Chin J Cancer 34: 1-3, 2015.

32. Schiemann WP, Blobe GC, Kalume DE, Pandey A and Lodish HF: Context-specific effects of fibulin-5 (DANCE/EVEC) on cell proliferation, motility, and invasion. Fibulin-5 is induced by transforming growth factor-beta and affects protein kinase cascades. J Biol Chem 277: 27367-27377, 2002.

33. Li H, Guan H, Guo Y, Liang W, Liu L, He X, Ke W, Cao X, Xiao H and Li Y: CITED1 promotes proliferation of papillary thyroid cancer cells via the regulation of p21 and p27. Cell Biosci 8: 57, 2018.

34. Dongre A and Weinberg RA: New insights into the mechanisms of epithelial-mesenchymal transition and implications for cancer. Nat Rev Mol Cell Biol 20: 69-84, 2019.

35. Lee YH, Albig AR, Regner M, Schiemann BJ and Schiemann WP: Fibulin-5 initiates epithelial-mesenchymal transition (EMT) and enhances EMT induced by TGF-beta in mammary epithelial cells via a MMP-dependent mechanism. Carcinogenesis 29: 2243-2251, 2008.

36. Pastushenko I and Blanpain C: EMT transition states during tumor progression and metastasis. Trends Cell Biol 29: 212-226, 2019.

37. Wang W, Chen H, Gao W, Wang S, Wu K, Lu C, Luo X, Li L and Yu C: Girdin interaction with vimentin induces EMT and promotes the growth and metastasis of pancreatic ductal adenocarcinoma. Oncol Rep 44: 637-649, 2020.

38. Chong GO, Jeon H-S, Han HS, Son JW, Lee YH, Hong DG, Lee YS and Cho YL: Differential microRNA expression profiles in primary and recurrent epithelial ovarian cancer. Anticancer Res 35: 2611-2617, 2015.

39. Llauradó M, Majem B, Altadill T, Lanau L, Castellví J, Sánchez-Iglesias JL, Cabrera S, De la Torre J, Díaz-Feijoo B, Pérez-Benavente A, et al: MicroRNAs as prognostic markers in ovarian cancer. Mol Cell Endocrinol 390: 73-84, 2014.

40. Xu W, Liu M, Peng X, Zhou P, Zhou J, Xu K, Xu H and Jiang S: miR-24-3p and miR-27a-3p promote cell proliferation in glioma cells via cooperative regulation of MXI1. Int J Oncol 42: 757-766, 2013.

This work is licensed under a Creative Commons Attribution-NonCommercial-NoDerivatives 4.0 International (CC BY-NC-ND 4.0) License. 